\title{
EDITORIAL \\ Early lessons in the management of COVID-19 for the pediatric neurosurgical community from the leadership of the American Society of Pediatric Neurosurgeons
}

\author{
John C. Wellons III, MD, MSPH, ${ }^{1}$ Gerald Grant, MD, ${ }^{2}$ Mark D. Krieger, MD, ${ }^{3}$ John Ragheb, MD, ${ }^{4}$ \\ Shenandoah Robinson, MD, ${ }^{5}$ Bradley Weprin, MD, ${ }^{6}$ and Jeffrey Ojemann, MD ${ }^{7}$ \\ ${ }^{1}$ Division of Pediatric Neurosurgery, Vanderbilt University Medical Center, Nashville, Tennessee; ${ }^{2}$ Department of Neurosurgery, \\ Lucile Packard Children's Hospital Stanford, Palo Alto, California; '3Department of Neurosurgery, Children's Hospital Los \\ Angeles, California; ${ }^{4}$ Department of Neurosurgery, Nicklaus Children's Hospital and the University of Miami, Florida; \\ ${ }^{5}$ Department of Neurosurgery, Johns Hopkins University School of Medicine, Baltimore, Maryland; ${ }^{6}$ Department of Neurosurgery, \\ University of Texas, Southwestern Medical Center, Dallas, Texas; and 'Department of Neurosurgery, Seattle Children's Hospital, \\ Seattle, Washington
}

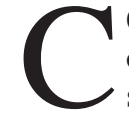

OVID-19 presents an unprecedented challenge to our population, our health care system, and our society. Several of our colleagues who hold leadership positions in their respective children's hospitals now have experience in preparing their local health care systems for COVID-19. It is our desire to summarize relevant experiences that they and their hospitals have had preparing for the challenges that COVID-19 presents. While the issues are legion, the topics that follow here are deemed the most crucial, and are listed as recommendations based on experience alone, as data for the degree of effectiveness are not yet available. One key theme across nearly all involved institutions has been the lack of severe illness in children to date, and to not let that lull the pediatric community into a lack of urgency in preparation and response.

\section{Children's Hospitals}

Travel for business and personal reasons has been and should remain limited. Access to entrances into the hospital should be restricted to 1-2 entrances, with a temperature screening and a series of symptom questions. Visitors for pediatric patients should be reduced to one adult for wards or clinics, with special permission required to rotate the visitor during the hospital stay. Many adult hospitals have begun a no-visitation policy. Surgical case prioritization rapidly came to the forefront of discussion due to disruptions in supply chains and the need for conservation of resources. The degree of case delay should be considered at individual institutions based on these issues. The initial dichotomy of elective versus emergent surgery was quickly found to be inadequate, as the majority of subspecialties and cases began to be prioritized based on the level of harm over the anticipated delay in care. There has been an unprecedented shift to telemedicine in the past 7 days, with IT departments and home internet providers straining to meet the need. How the pediatric neurosurgery clinic is able to be conducted remotely is yet to be fully experienced, but the early experience is positive. Many nonessential visits and/or imaging procedures have been pushed ahead by several months. Federal and state laws have rapidly evolved to meet the need for telemedicine licensure and billing; this is encouraged, and a long-term impact on pediatric neurosurgical follow-up protocols is anticipated. Social distancing for clinical and administrative meetings using videoconferencing platforms such as Zoom, Skype, or others should be implemented. Lastly, conservation of personal protective equipment (PPE) should be in full effect.

\section{Operating Rooms}

We should respect airway management protocols for intubation/extubation by the anesthesiologists. Seattle Children's Hospital, an institution involved with the COVID-19 response early on and hence is 1-2 weeks ahead of most institutions, has recently instituted a policy in which all patients will be intubated with minimal personnel in the room, using contained air purifying respirators. Then, 30 minutes must pass before the rest of the operating room (OR) personnel enter the room. After extubation, 30 minutes must also pass before cleaning crews enter, all done to reduce viral particulate matter exposure. These rules will be relaxed for non-gastrointestinal cases once rapid testing is available and patients test negative for COVID-19. Other centers are now adopting similar protocols with variable out-of-room wait time for non-airway personnel after airway manipulation. Additional precau- 
tions noted include limiting personnel to only those necessary for the procedure to be performed safely. It should be noted here that viral load is considered to be high in the nose and nasopharynx, and the risk to the surgical team is notable for endonasal surgery. Many places with rapid COVID-19 testing turnaround are mandating testing prior to proceeding with endonasal surgery of any type. Those with longer testing turnaround time are faced with identifying alternative surgical treatment strategies and stratifying the degree of urgency. Several centers have recently experienced a significant reduction in turnaround time for COVID-19 testing, down to 8 hours. OR policy must therefore be made around the most rapid testing available at each institution. In terms of resource utilization due to supply-line issues, some institutions have limited the use of disposable products and are switching to reusable cloth drapes and gowns.

\section{Pediatric Neurosurgery Clinical Team}

Clinical team-based rotations should begin in an effort to reduce virus exposure and conserve team members for clinical care. Most medical student rotations have been cancelled and these students sent home for online learning. Residents and advanced practice providers should be on limited contact hours and be at home when not working. Social distancing for all group-based activities should be taking place, and rounds should be conducted with as minimal personnel as possible using the appropriate protective gear. Our field, by nature, lends itself to long hours and teamwork. We will need to "lean out to lean in," to go home at scheduled intervals, and embrace the shift worktype mentality that we have been reluctant to follow until now. Policies around pregnant workers are needed.

\section{Patients}

Many patients are appreciative of these measures taken in the outpatient setting and are agreeing to delayed follow-up or the transition to telehealth. In the inpatient area, there has been some experience early on with patients and families minimizing flu-like symptoms in order to have their less urgent surgery performed, which should be ameliorated by case prioritization and more stringent screening processes. Many of our patients with "chronic" pediatric neurosurgical issues involve intermittent screening, either by visit and/or imaging, as well as heightened vigilance for life-threatening conditions. Sedation imaging requiring airway control could put anesthesia providers at risk and should be screened for urgency as well. It is of the utmost importance to reassure both our patients and their families that while these policies are in place to protect them and their providers, our teams remain available to them in emergencies just as before. Therefore, we must ensure that our ability to triage by phone has never been better.
We must be prepared to push our way out of our comfort zones even further. We must be cognizant of our adult colleagues and work to ensure adequate coverage of emergency cases as the number of available staff is reduced. These are even more reasons to do everything within our power to reduce the likelihood of exposure and infection by following the recommendations based on combined experience above. Unless we see a shift in illness burden to children, we may need to be prepared to loan out our pediatric ventilators. Some institutions may even be called upon to transfigure some portion of the pediatric intensive care units, recovery beds, and even ORs to be used for adult critical care, all while allowing us to take care of the needs of the children of our community, which is a critical balance to strike. Whether or not as subspecialists we will be pulled in the most extreme circumstances to ventilator management, front-line screening, or palliative care as reported elsewhere, remains to be seen.

One thing is for certain. Pediatric neurosurgery is a field composed of resilient, hardworking women and men who have dedicated their lives to the care of others. We have sat with our patients and parents and delivered the hard news. We have spent the extra time under the microscope taking one more look around the fourth ventricle and found that little bit of tumor out in the recess that had been hiding under the retractor blade. We have learned from our colleagues of all ages new techniques to keep up with modern treatment paradigms, and we have pushed ourselves and our field forward by embracing challenge and change. Our medical world has arguably evolved more rapidly in the past week than in modern history. We look back to the lessons and leaders of the past for steady guidance, and we will do what we are called to do firmly with the knowledge that one day, perhaps decades or even centuries from now, humanity will look back to us for the same.

https://thejns.org/doi/abs/10.3171/2020.3.PEDS20215

\section{Disclosures}

The authors report no conflict of interest.

\section{Correspondence}

John C. Wellons III: jay.wellons@vumc.org.

\section{INCLUDE WHEN CITING}

Published online April 10, 2020; DOI: 10.3171/2020.3.PEDS20215. 